\title{
RoSe: A Subgigahertz Wireless Sensor Platform With Housing-Integrated Overmolded Antenna
}

\author{
Mitko Tanevski, Graduate Student Member, IEEE, Francesco Merli, Member, IEEE, Alexis Boegli, \\ Anja K. Skrivervik, Jean-François Zürcher, and Pierre-André Farine, Member, IEEE
}

\begin{abstract}
A wireless sensor platform for use in ultralow-power wireless sensor networks (WSNs) is presented. The proposed round sensor (RoSe) node is physically robust, is resistant to humidity, and has a housing that can be in direct contact with food. These characteristics are achieved via injection molding technology which is investigated at both the component and the system levels. The results of injection molding tests on the node's electronic components are reported, while a housing-integrated overmolded antenna is conceived and realized. The radiator, operating in the subgigahertz industrial, scientific, and medical (ISM) band (ISM 868 or $915 \mathrm{MHz}$ ), has been characterized, and a good correspondence with theoretical predictions is observed. The proposed system enables industrialized mass production and results in a generic platform for wireless monitoring applications. In this paper, we present the RoSe node with a temperature sensor for use in food cold chain management, i.e., for use at temperatures down to $-30{ }^{\circ} \mathrm{C}$ and in frequent contact with water due to condensation or cleaning. Its ultralow-power characteristics are demonstrated by the analysis of the charge consumption when the node performs specific tasks both in a test case and operating in a WSN. Long-term battery lifetime results while running a demo WSN are also presented.
\end{abstract}

Index Terms-Food compatibility, long battery autonomy, overmolded antenna, subgigahertz robust wireless sensor platform, temperature monitoring, ultralow-power consumption, wireless sensor networks (WSNs).

\section{INTRODUCTION}

$\mathbf{T}$ HE INTEREST toward wireless sensor networks (WSNs) remains ever growing. Standard protocols [1], [2] have been defined, and several solutions have been introduced in the last decade [3]-[9].

Most of the mentioned published research describes either bare node platforms as in [3] and [4] or node platforms in application-specific prototype housing [5]-[7]. In [5], for instance, the authors present a modular approach built around the battery accepting it as the largest part of the wireless sensor

Manuscript received December 21, 2011; revised March 22, 2012; accepted March 23, 2012. Date of publication July 27, 2012; date of current version October 10, 2012. This work was supported in part by the Swiss Confederation's innovation promotion agency-Commission for Technology and Innovation-under reference CTI ROSE 8711.2. The Associate Editor coordinating the review process for this paper was Dr. Deniz Gurkan.

M. Tanevski, A. Boegli, and P.-A. Farine are with the Electronics and Signal Processing Laboratory, École Polytechnique Fédérale de Lausanne, 2000 Neuchâtel, Switzerland (e-mail: mitko.tanevski@epfl.ch).

F. Merli, A. K. Skrivervik, and J.-F. Zürcher are with the Laboratory of Electromagnetics and Acoustics, École Polytechnique Fédérale de Lausanne, 1015 Lausanne, Switzerland.

Color versions of one or more of the figures in this paper are available online at http://ieeexplore.iee.org.

Digital Object Identifier 10.1109/TIM.2012.2202184 node and using a commercial off-the-shelf monopole antenna of acceptable size. In [6], the authors built a waterproof packaging and developed their protocol for a node based on a standard electronics for the application of debris flow monitoring. In [7], the platform presented uses a node smaller in size than that in [6] and provides much greater autonomy. Indeed, the terms low power and energy efficient translate in different battery autonomies for distinct applications having 5 months on 2-D-size batteries in [6] and targeting more than 2 years on CR2477 primary coin cell in [7]. Proprietary system-on-chip solutions [8] have also been proposed by combining the microcontroller (MCU) and the transmitter on a single chip.

This paper, extending the results presented in [10], focuses on a new physically robust and ultralow-power wireless sensor platform. The platform benefits from a novel concept of antenna housing which provides both electrically robust and efficient antennas as well as housing which is mechanically robust and resistant to humidity. Such a design has been made possible by integrating electromagnetic (EM) aspects with injection molding technology [11], which is compatible with highvolume and low-cost industrial production. Given the everincreasing interest toward this technological process, this paper also discusses the overmolding of the active electronic components. The design and realization of each element constituting the node (electronics hardware, antenna housing, battery, and communication protocol) have been carefully investigated to reach a compact highly integrated device. Particular attention was paid to the node energy consumption characterization, so as to enable the evaluation of the round sensor (RoSe) node for different internal power supplies, WSN applications, and protocols.

This paper is organized as follows: Section II describes the architecture of the RoSe node, while Section III reports the injection molding tests performed on the electronics. The development of the housing-integrated overmolded antenna is presented in Section IV along with the radiator experimental evaluation in terms of both materials characterization and radiation performances. Section V proposes a per-task charge consumption measurement method to evaluate the charge requirements of the node and communication protocol and gives the results from long-term battery lifetime measurements. Finally, conclusions are drawn in Section VI.

\section{RoSe Node ARchitecture}

The RoSe wireless node is a complete wireless platform composed of several elements, namely, MCU, quartz crystal 


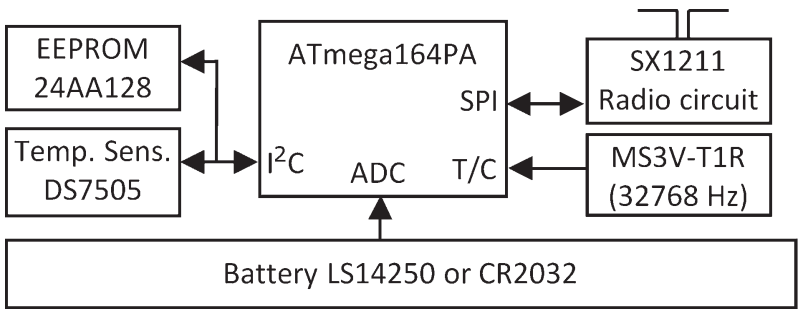

Fig. 1. Schematic showing the main components of the RoSe node architecture. The reference design of SX1211 was implemented [13].

for the asynchronous timer, electrically erasable programmable read-only memory (EEPROM), radio transceiver, antenna, power supply, and temperature sensor. As each of them comes with its own requirements and specifications, a global approach was used in order to reach the highest level of integration and miniaturization. The housing-integrated overmolded antenna detailed in Section IV allows for the placement of all the electronics components in a circular printed cicuit board (PCB) without limiting their choice (for instance, to system-on-chip solutions). Therefore, an exhaustive survey of commercially available elements was performed so as to identify the best tradeoff in terms of energy efficiency, price, and tools for development.

Fig. 1 describes the schematic, selected components, and connections of the RoSe node architecture. The Atmel picopower MCU ATmega164PA MCU was chosen as it offers firmware scalability and all the advantages of a mainstream MCU in terms of development and production tools. Furthermore, the MCU's internal analog-to-digital converter (ADC) enables the monitoring of the battery voltage. The radio transceiver of the platform is Semtech SX1211, which has a digital part with a buffer and a minimum operating voltage of $2.1 \mathrm{~V}$, allowing for $3-\mathrm{V}$ primary cells to be used. It also has a low current consumption of $3 \mathrm{~mA}$ in reception mode. The node architecture allows for two kinds of batteries to be used: one 1/2 AA or two CR2032. Additionally, a supercapacitor of $75 \mathrm{mF}$ (CAP-XX GZ215F) can be mounted to extend the usable charge of the batteries by minimizing the supply voltage drop during the transmission current peaks. The Microchip 24AA128 EEPROM and the Maxim DS7505 temperature sensor are both connected to the two-wire interface (i.e., $\mathrm{I}^{2} \mathrm{C}$ bus [12]; hence, other memories and sensors can be used, requiring minimal hardware intervention). Fig. 2(a) shows the node's components mounted on the top side of a circular PCB. This solution results in a simple production process and allows the placement of the battery holder on the bottom side of the same PCB as shown in Fig. 2(b). Exposed pads are also placed next to the battery connectors for production programming and testing purposes. The pads also enable the use of wired power supply and data connections providing a node which can be used for different roles in a WSN (relay, gateway, etc.). A total of 20 PCBs were produced, mounted, and tested, verifying the repeatability of the production process.

\section{Tests of InJeCtion Molding}

In order to protect the PCBs from the humidity of highcondensation environments (such as refrigerators), we first ex-

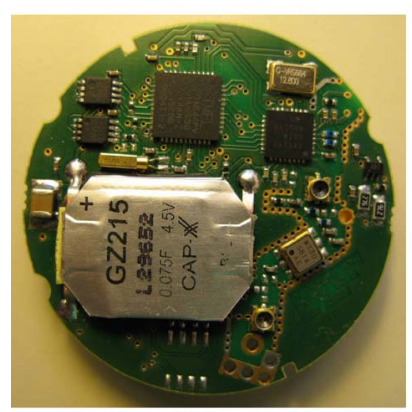

(a)

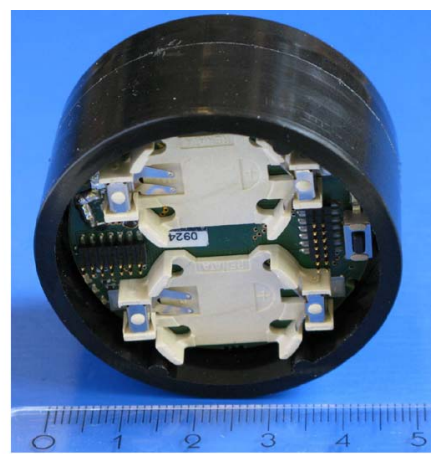

(b)
Fig. 2. RoSe prototype. (a) The PCB with the electronic components (b) Bottom view of the complete node showing the back side of the PCB mounted inside the antenna housing.

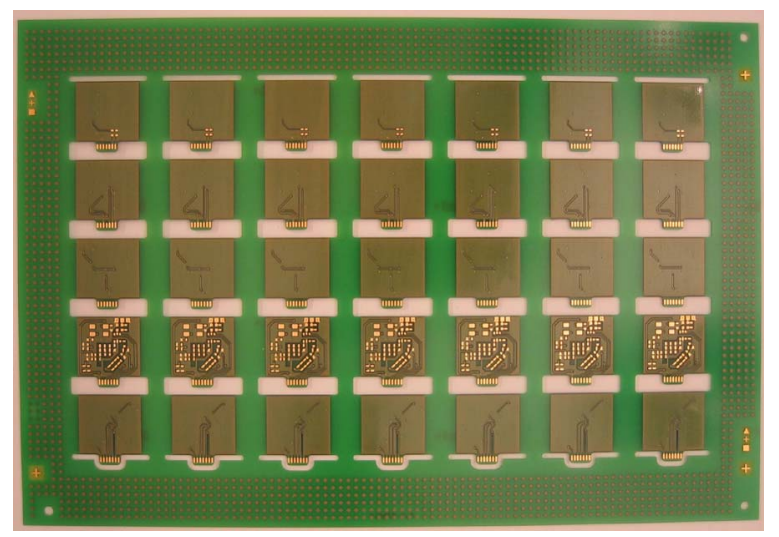

Fig. 3. PCBs for injection molding component testing.

plored the possibility of overmolding the electronics hardware. This process is suitable for high-volume production, and it provides both the resistivity to humidity and the required food compatibility given a proper choice of the plastic material (polypropylene for our application). According to the authors' best knowledge, injection molding complex electronic circuits with polypropylene have not been investigated and had to be performed. Injection molding, which is widely used in the mass production of plastic parts, involves the injection of a polymer (polypropylene in our case) into the mold at a high temperature and pressure of approximately $220^{\circ} \mathrm{C}$ and $800 \mathrm{bar}$, respectively. These harsh conditions, although short in duration, can compromise the integrity of the $\mathrm{PCB}$ and of its components. Temperature alone is not the main concern in this case because soldering is performed at similar or higher temperatures and longer durations. However, manufacturers of the electronic components that were used do not provide any information about the pressure. Furthermore, during the injection process, the flow of the polypropylene under pressure could cause damage to the components or detach them from the PCB. In order to verify the capability of the electronic components to survive such conditions, several subcircuits, shown in Fig. 3, were tested separately by measuring their specified parameters before and after the injection molding process. The analysis of the PCBs after injection molding, shown in Fig. 4, immediately showed that the MCU was still fully functional (the device was reprogrammable, and the test program was 


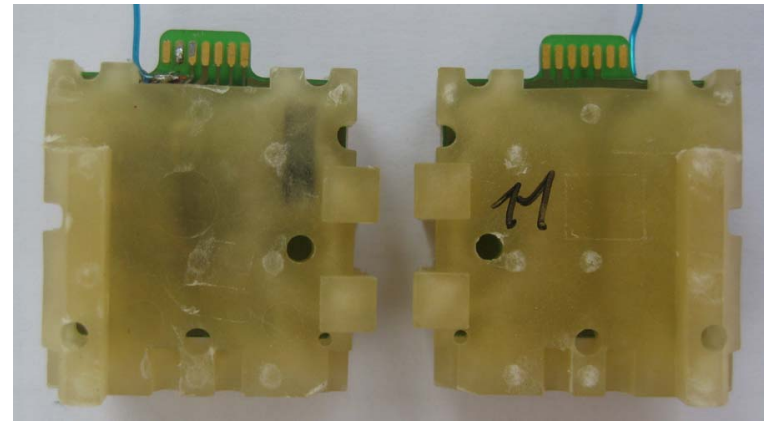

Fig. 4. Two identical PCBs after injection molding (front and back views).

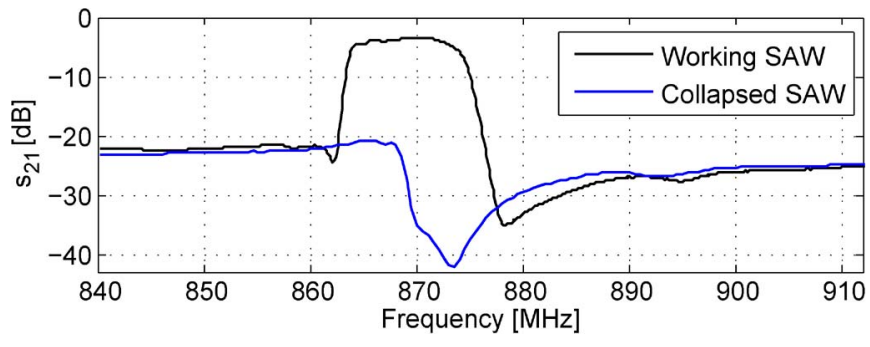

Fig. 5. $s_{21}$ parameter for a functioning SAW and collapsed SAW measured with the same setup, using Agilent E8358A RF performance network analyzer.

running, flashing the mounted LEDs which are visible through the mold). Furthermore, all components remained attached to the PCBs and maintained the desired electrical connections. Two types of 32-kHz quartz-crystal frequency oscillators were mounted on the test PCBs: the Micro Crystal MS3V-T1R and the Citizen C2KC57ES. No modifications were observed for the first crystal, whereas the frequency deviation of the C2KC57ES was in the order of several parts per million (ppm). Considering that both crystal types have production deviations of $\pm 20 \mathrm{ppm}$ and that all 25 tested samples were still oscillating after the test, we concluded that the injection molding process of the $32-\mathrm{kHz}$ crystals posed no problems.

On the other hand, the surface acoustic wave (SAW) filter and the 12.8-MHz quartz crystal for the radio transceiver chip were damaged by the injection molding to a nonfunctional state. The conducted measurements of the SAW filter using a two-port network analyzer after injection molding showed that the component is not working (i.e., no signal got through it-Fig. 5). Likewise, the $12.8-\mathrm{MHz}$ quartz did not provide any oscillating output when measured by a universal counter. The origin of such damages is the mechanical deformation of these components produced by the injection molding process as shown in Fig. 6. These two components have partially hollow housings closed with a large top surface which collapses toward the inside of the component because of the high pressure of the injection molding process. The problem can be mitigated by applying a resin before the overmolding as shown in Fig. 7. Indeed, the SAW filter covered with resin survived the injection molding process showing no changes in the measured parameters as compared to prior the injection molding. By performing these injection molding tests, we proved that overmolding complex electronics is possible, and, taken some precautions, a node would retain its full functionality. On the contrary, the complete injection molding of a device would deny the possibility of

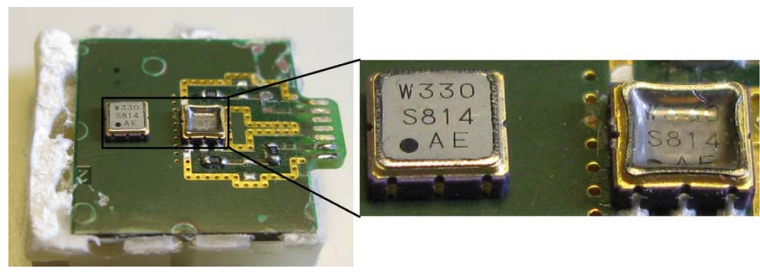

Fig. 6. View on the collapsed SAW filter after removing the plastic mold. A new SAW filter is placed on the board for comparison. Similar mechanical deformations were observed for the $12.8-\mathrm{MHz}$ transceiver quartz oscillator.

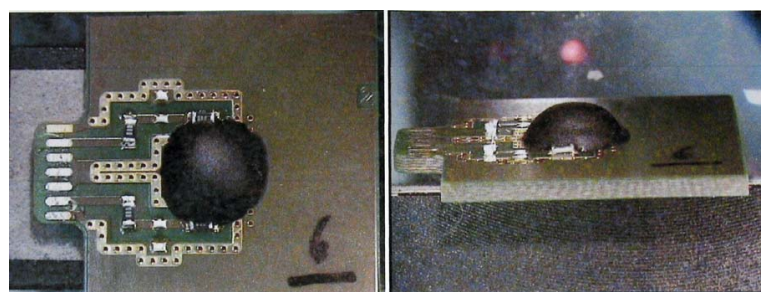

Fig. 7. Resin applied over the SAW before the injection molding.

replacing the battery (in addition to the harsh conditions that could affect its proper working behavior). Therefore, the concept of housing-integrated overmolded antenna was developed to provide a food-compatible robust housing to the entire node without limiting the access to the power supply.

\section{HOUSING-INTEGRATED OVERMOLDED ANTENNA}

\section{A. Conception}

When targeting a wireless node in the subgigahertz frequency range, the antenna is usually one of the most demanding and limiting factors for the overall miniaturization (the battery being the only possible exception). In this paper, a compact 3-D cylindrical antenna was designed to optimally integrate with the electronics and the power supply of the RoSe node.

Based on the planar inverted-F antenna (PIFA) typology [14], the new radiator combines a thick planar section with a 3-D cylindrical ground plane, as shown in Fig. 8. The relatively large distance $(8.4 \mathrm{~mm})$ between the patch and the ground plane is realized with the use of three dielectrics. A plastic layer of yellow polypropylene, realized with injection molding technology, is inserted between the two single-side FR4 substrates supporting the printed patch and the ground, respectively. Finally, the entire radiator is overmolded by a black polypropylene layer. The top view of the radiating patch is shown in Fig. 9 which shows the two slots etched to reduce the resonant frequency to the desired $868-\mathrm{MHz}$ industrial, scientific, and medical (ISM) band and the ground connection realized via four metallic stiffs. The proposed design fulfils EM, mechanical, mass production, and food-compatible requirements. First, the efficient use of the available volume enhances both the miniaturization of the radiator itself (maximum dimension comparable with $\lambda / 7$ ) and the radiation performance (efficiency). Furthermore, only a shortening of the patch length (without changing neither the excitation points nor the rest of the antenna geometry) ensures its use in the U.S. ISM $915-\mathrm{MHz}$ frequency spectrum. Second, the design provides a mechanically robust housing which also serves as an efficient shielding 


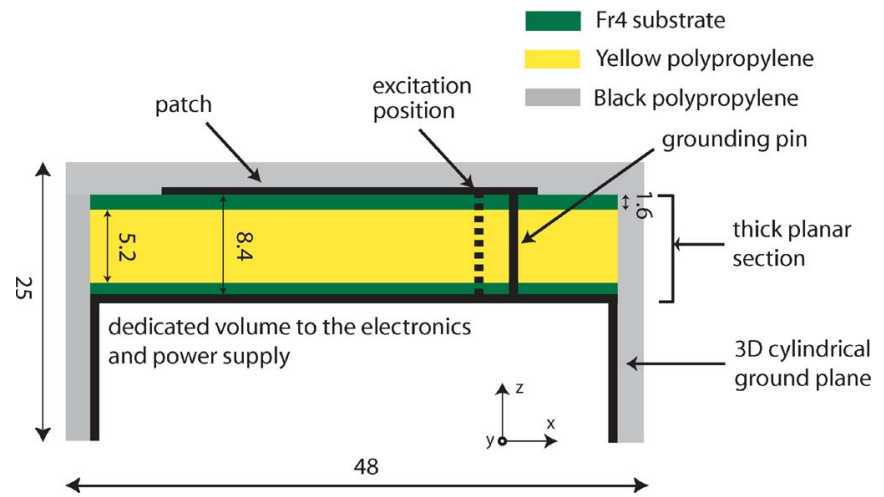

Fig. 8. Sketch of the realized antenna (cross section). The solid black lines represent metallic structures, while the dashed line indicates the excitation position. The grounding pin short-circuits the patch and the ground parts as in PIFAs so as to facilitate the antenna miniaturization. Dimensions are in millimeters.

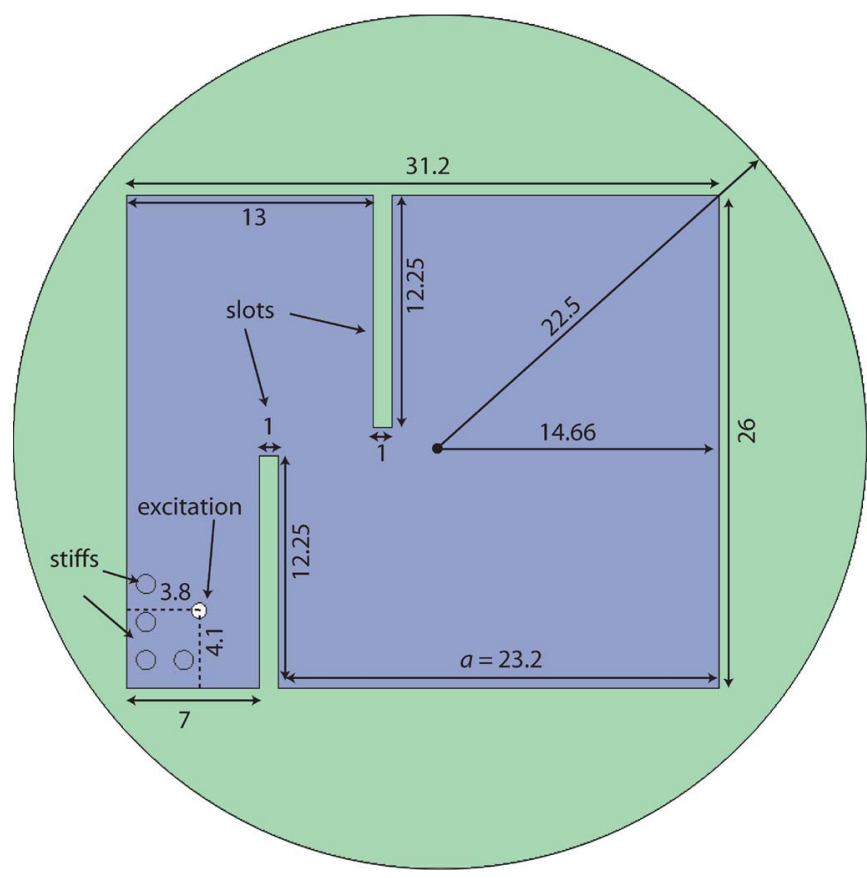

Fig. 9. Top view of the patch of the overmolded antenna (units in millimeters). The grounding pin connection between the patch and the ground plane is realized via four metallic stiffs. Reducing the length $a$ from 23.2 to $20.5 \mathrm{~mm}$ shifts the working frequency from $868 \mathrm{MHz}$ to the U.S. ISM 915-MHz band.

for the electronics. In fact, the PCB, once mounted, is protected from undesired external EM interferences by the cylindrical ground plane. Finally, polypropylene substrates provide the required food compatibility and resistivity against humidity and ensure the good reliability of the production. Indeed, the high precision and the versatility of the overmolding process can counterbalance the standard PCB fabrication tolerances.

\section{B. Dielectric Measurements}

Since the use of an overmolded polypropylene material truly belongs to the antenna concept, its precise dielectric characterization is mandatory for a successful realization. Keeping in mind that polypropylene is mixed with other materials (such as colorant, fiber glass, etc.) during the injection molding

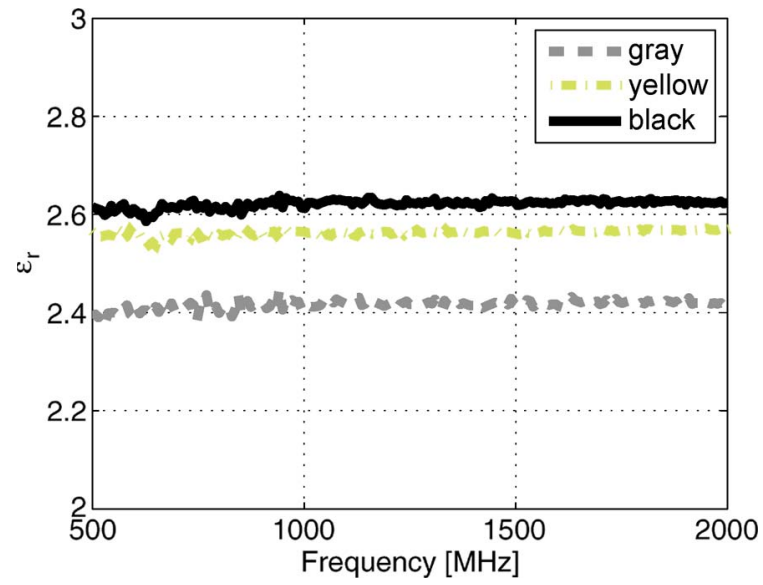

Fig. 10. Measured $\varepsilon_{r}$ values of polypropylene materials of different colors after the overmolding process (uncertainty of about 5\%). The averages of the measurements of several material samples are considered.

TABLE I

Measured Dielectric Properties of Different OVERMOLding Plastics AT $868 \mathrm{MHz}$

\begin{tabular}{c|c|c}
\hline \multirow{2}{*}{ Material } & \multicolumn{2}{|c}{ Dielectric properties } \\
\cline { 2 - 3 } & $\varepsilon_{r}$ & $\tan \delta$ \\
\hline \hline $\begin{array}{c}\text { Gray Polypropylene } \\
\text { after overmolding }\end{array}$ & 2.44 & $\leq 0.006$ \\
\hline $\begin{array}{c}\text { Yellow polypropylene } \\
\text { after overmolding }\end{array}$ & 2.55 & $\leq 0.003$ \\
\hline $\begin{array}{c}\text { Black polypropylene } \\
\text { after overmolding }\end{array}$ & 2.63 & $\leq 0.007$ \\
\hline \hline
\end{tabular}

process, several plastic samples have been tested over a wide frequency range using the HP Dielectric Probe Kit [15] and the transmission line method [16]. The measured relative dielectric constants $\varepsilon_{r}$ for different colors are shown in Fig. 10. A relative difference up to almost $+8 \%$ was found between the black and gray samples. The difference is even larger, i.e., $\simeq 15 \%$, if compared to pure polypropylene (nominal $2.25 \leq \varepsilon_{r} \leq 2.36$ [17]). Table I reports the measured dielectric characteristics at $868 \mathrm{MHz}$ which were used during the numerical investigations of the antenna. As for the relative dielectric constant, the use of colorants increases the losses in pure polypropylene (nominal $\tan \delta \leq 0.002$ [17]). Given the properties reported in Table I, gray polypropylene was discarded as it presents the lowest $\varepsilon_{r}$ (thus, not facilitating the miniaturization of the radiator), while the yellow and the black ones were used for the internal substrates and the encapsulation of the radiator, respectively.

\section{Realization and Measurements}

The fabrication of the proposed antenna consists of three steps. First, the metallization on circular FR4 PCBs is realized via standard etching procedures. Second, the metallic stiffs and the cylindrical ground plane were manufactured in brass. Finally, overmolding with black polypropylene was performed on the antenna assembly including the internal yellow polypropylene spacer. A realized antenna with an assembled electronics is shown in Fig. 2(b). 


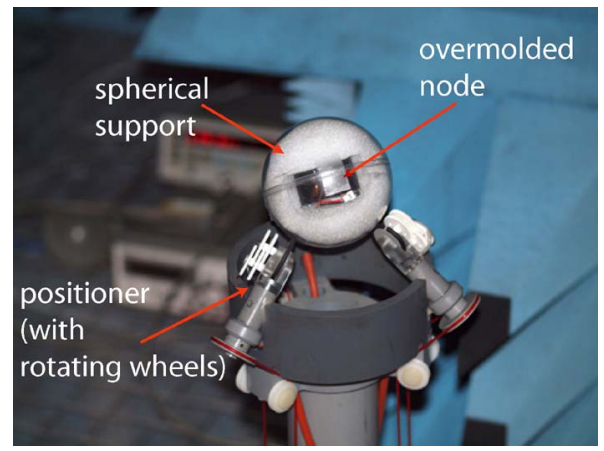

Fig. 11. Complete node integrating the antenna, the electronics, and the power supply in the anechoic chamber. The antenna (overmolded with black polypropylene) is enclosed in a spherical support whose rotation (owing to the nonmetallic "positioner") allows the measurement of the radiation over the entire solid angle $(4 \pi)[18]$.

The small electrical size of the radiator implies several difficulties when testing its performances [18], [19]. The radiation characteristics were measured with the antenna integrated with the developed electronics. Following [18], the complete node was inserted in a spherical support which is consequently rotated by a nonmetallic "positioner." The maximum realized gain is evaluated by measuring the received signal by a reference antenna $(\lambda / 2$ dipole), after the characterization of the power emission of the integrated generator. In fact, the rotation ensures the evaluation of all different orientations between the node and the reference antenna. Fig. 11 shows the measurement setup in an anechoic chamber. Radiation patterns and realized gain (including mismatching losses) are shown in Figs. 12 and 13 , respectively, obtaining satisfactory agreement with the expected results. An upward frequency shift $(5 \mathrm{MHz})$ of the measured performances compared to the simulated ones is observed because of fabrication imperfections. A maximum gain difference of $0.5 \mathrm{~dB}$ is registered, which is within measurement tolerances. The maximum gain values are low because of the quite high losses of the used low-cost materials (FR4 substrates, brass, and overmolded polypropylene). Mismatching losses are not remarkably important as simulated results consider a good matching (e.g., $\left|s_{11}(f)\right|<-20 \mathrm{~dB}$ at $869 \mathrm{MHz}$ on a $50-\Omega$ load). The agreement of the simulations and measurements of the realized gain also validates the matching characteristics.

A total of 15 antenna housings were produced, and 15 of the tested PCBs were mounted inside the housing [Fig. 2(b)]. A back-side enclosing part was also constructed through injection molding in order to demonstrate the bayonet enclosure as shown in Fig. 14. By using an O-ring, the complete enclosure becomes waterproof. This packaging, together with the housing-integrated overmolded antenna, provides a robust solution without requiring electronics overmolding. Outdoor range measurements were performed to validate the systemlevel functionality of the assembled node in line-of-sight conditions. A test node was programmed to send test frames, while a second one was programmed to stay in reception mode and report received packets, errors, and signal levels. A measurement along a suitable part of the shore of Lake Neuchâtel in Switzerland was performed. The distance was increased until transmission errors started appearing. The achieved range with

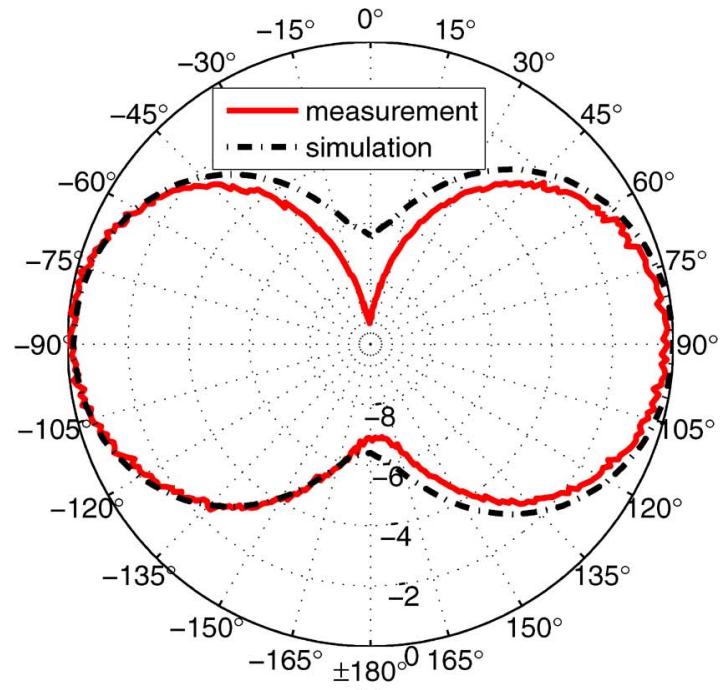

(a)

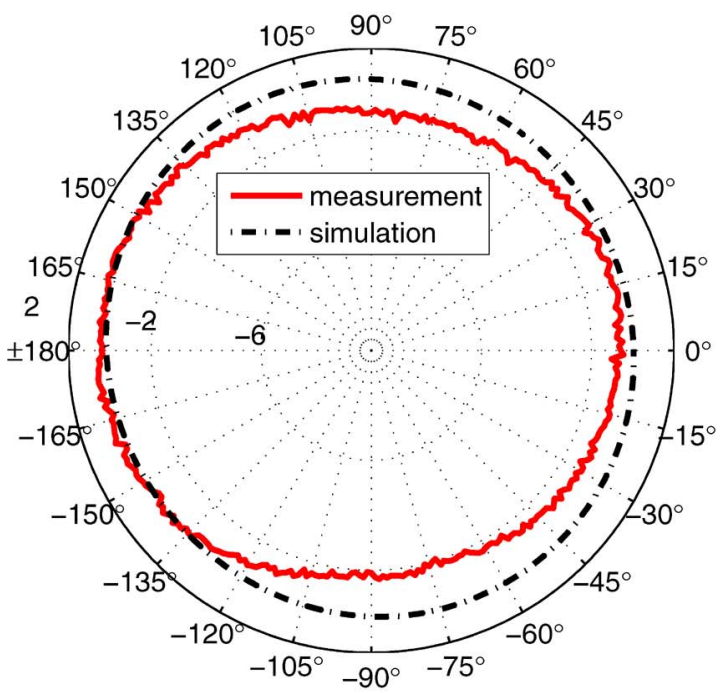

(b)

Fig. 12. Measured and simulated radiation patterns: (a) Vertical plane $(z x)$ and (b) and horizontal plane $(x y)$. Simulations are performed with ANSYS HFSS version 13 , and values are normalized to $0 \mathrm{dBi}$. The coordinate system is shown in Fig. 8.

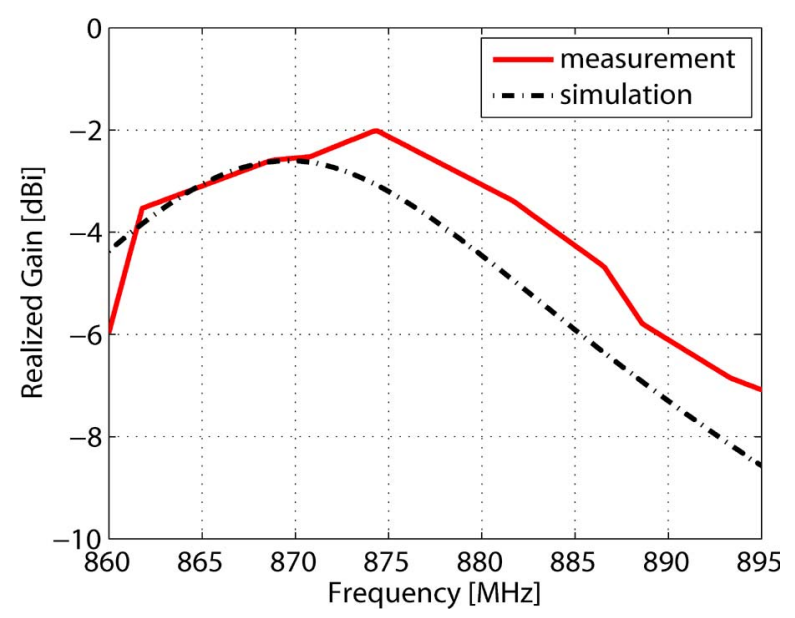

Fig. 13. Measured and simulated realized gains of the realized prototype. 


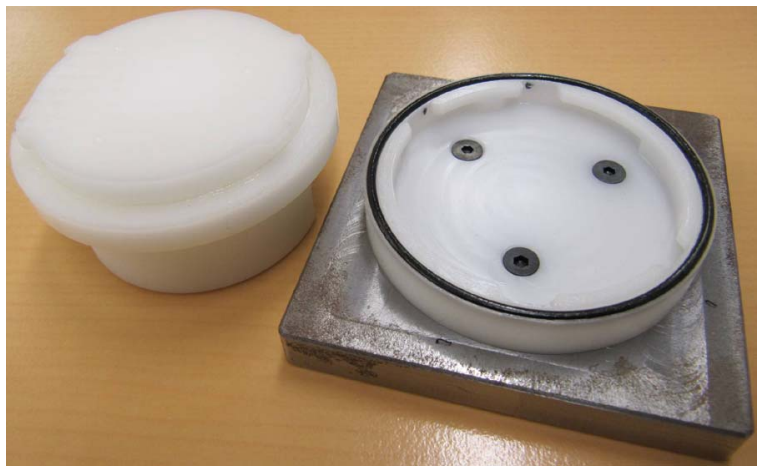

Fig. 14. Model for demonstration of the bayonet enclosure of the node including the O-ring.

the embedded antennas on both ends was approximately $650 \mathrm{~m}$ without transmission errors (using two-level frequency-shift keying (FSK) with a deviation of $\pm 50 \mathrm{kHz}, 25-\mathrm{kb} / \mathrm{s}$ bit rate, frame length of $64 \mathrm{~B}$, and maximum output power).

\section{RoSe Node IN A WSN}

In order to demonstrate the ultralow-power capability of the proposed node, let us discuss the WSN architecture and communication protocol, the energy consumption, and longterm battery lifetime. This analysis shows also how the RoSe platform outperforms (and aims at replacing) an existing node [20]. The latter is already running in several commercial industrial installations including a system for monitoring the temperature in the refrigerators in one supermarket chain in Switzerland. This application requires one measurement every 15 min which represents more than 1 million measurements every week, considering the whole network of more than 2000 sensor nodes.

\section{A. WSN Architecture and Communication Protocol}

The sensor node itself as a hardware platform is only one part of a specific WSN application such as the commercial system for monitoring the temperature. The core of the system is constituted of a WSN coordinator software and three different types of wireless and wired nodes: sensors, relays, and a gateway/bridge. The WSN coordinator software runs on a computer (or a server) and is responsible of several tasks such as the following: establishing and maintaining the network, storing measurements into a database, and retrieving measurements from nodes that have been temporarily disconnected. A frontend software connected to the database performs the alarming when the temperatures exceed their predefined limits. The gateway/bridge node makes the connection between the wireless and the wired medium, whereas the relay node extends the working range. While these two devices have an unconstrained power supply, the sensor node performs measurements and transmits data relying on the internal battery. This star-mesh WSN topology is shown in Fig. 15. The design and architecture of the proposed RoSe node provide a common hardware solution for all the three different nodes with the use of a specific firmware for each node type.

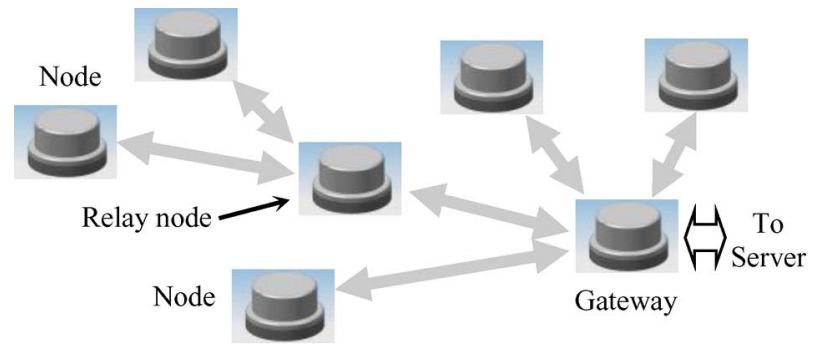

Fig. 15. Star-mesh network topology (note the absence of node-to-node communication).

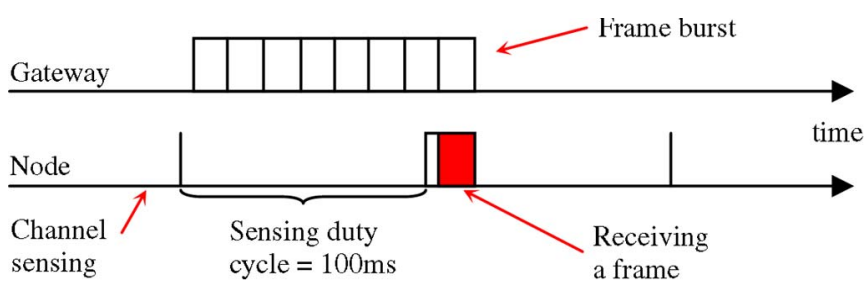

Fig. 16. Chronogram example of the frame bursting technique.

The commercialized application uses a proprietary communication protocol called INtact owned by IP01 SA [21], which was ported to the RoSe node MCU. This protocol, working in a star-mesh network topology, supports mobility (refrigerators are being moved, rearranged, or cleaned) and logs the measurements locally on an EEPROM in order to provide redundancy in case of communication failure. The radio and the physical layer parameters used in all the tests in this section are as follows:

1) modulation: two-level FSK with a deviation of $\pm 50 \mathrm{kHz}$;

2) bit rate: $25 \mathrm{~kb} / \mathrm{s}$ - no channel coding applied;

3) channel access method: carrier sense multiple accesscollision avoidance (CSMA-CA);

4) channel sensing criteria (free/busy): signal level (received signal strength indicator) value;

5) maximal frame size: $128 \mathrm{~B}$.

In order to save energy, the channel is sensed once every $100 \mathrm{~ms}$ by measuring the signal level. If sensed busy, the node remains in receiving mode, waiting for the start of a frame. To ensure reception, the sender must transmit multiple copies of the same frame in a burst with a total duration of more than $100 \mathrm{~ms}$ such that at least one complete frame is transmitted outside of the 100-ms period (shown in Fig. 16). The usual length for a measurement frame is $61 \mathrm{~B}$ which, at $25 \mathrm{~kb} / \mathrm{s}$, lasts $19.52 \mathrm{~ms}$; hence, the technique described in Fig. 16 implies transmission bursts of $136.6 \mathrm{~ms}$ and average frame receiving time of $29.3 \mathrm{~ms}$. To further increase energy savings, the node does channel sensing for $3 \mathrm{~s}$ after data transmission (with a duty cycle of $100 \mathrm{~ms}$ ) and subsequently goes to power-save mode until the scheduled time for the next measurement. In this way, the WSN coordinator can send a frame to a node only within $3 \mathrm{~s}$ after receiving one.

\section{B. Per-Task Charge Consumption Measurements}

Charge consumption evaluation was used for an immediate comparison with the battery ratings provided by the manufacturers. The supply current waveform was sampled and used 


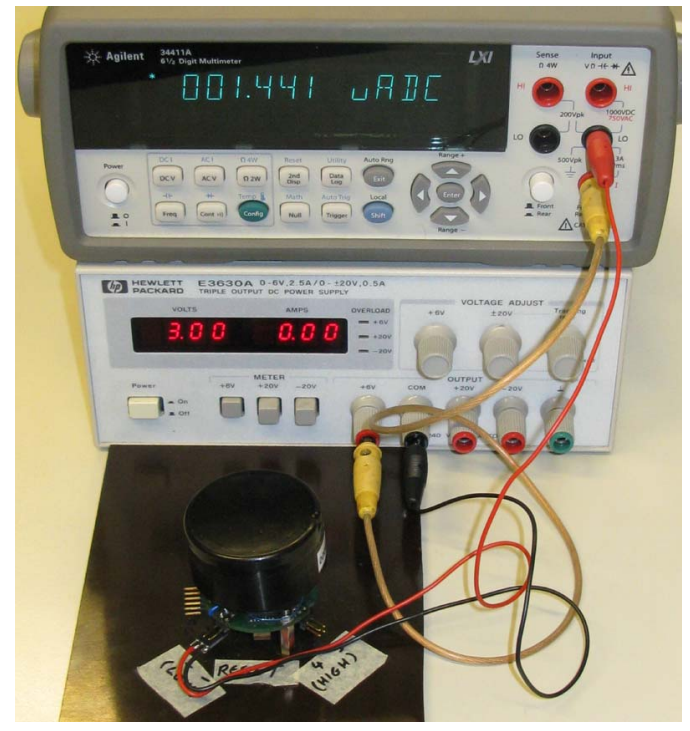

Fig. 17. Setup for supply current waveform acquisition. The node is placed on a board that also provides connections for programming and debugging.

to obtain the total electric charge consumed by the node for different tasks (such as the following: time keeping, channel sensing, frame transmission, frame reception, and temperature measurement). As the energy consumption varies slightly with the voltage (it increases at higher voltages and decreases at lower ones), a fixed 3-V value provided from the HP E3630A was used as it is approximately in the middle of the operating range of the node. All the measurements were performed using the Agilent 34411A 6 1/2 digit multimeter to sample the current at 50000 samples/s. The multimeter was connected via Universal Serial Bus (USB) to a PC which was running a MATLAB program for the waveform acquisition. Fig. 17 shows the measurement setup.

All charge consumption values in this section represent a mean value of at least three measurements considering two different cases: test (the node continuously runs only the specific task) and demo (the node belongs to a WSN-seven sensor nodes and one gateway node-when performing the task). Multiple measurements of consumed charge were performed, showing a maximum deviation (from the mean) of less than $0.3 \%$ for each task on each single node. For the transmission and reception tasks, the maximum deviation between different nodes was up to $7.1 \%$ which is due to production variations in the radio circuit.

1) Time Keeping: Each node has an asynchronous timer enabled at all times in order to keep an internal clock. When in power-save mode, the node requires a supply current ranging from 1.44 to $1.62 \mu \mathrm{A}$. A time-keeping interrupt wakes up the node each second in order to update the clock. Fig. 18(a) shows the supply current waveform for a clock timer update in the test case, while the demo one is shown in Fig. 18(b). The latter situation implies that the interrupt executes more code in order to update some protocol counters and to check if any conditions are met for further action. The charge consumed for the clock and protocol timers update for $50 \mathrm{~ms}$ after the current rise is $197 \mathrm{pAh}$. The maximum power-save current is $1.62 \mu \mathrm{A}$ which, for $50 \mathrm{~ms}$, represents $22.5 \mathrm{pAh}$. Hence, updates of the clock and

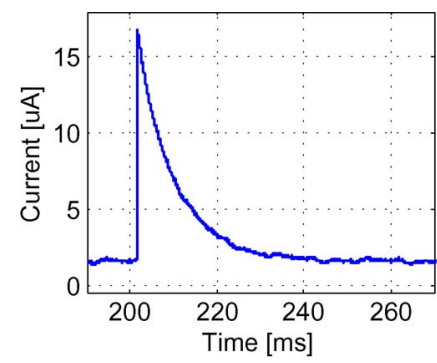

(a)

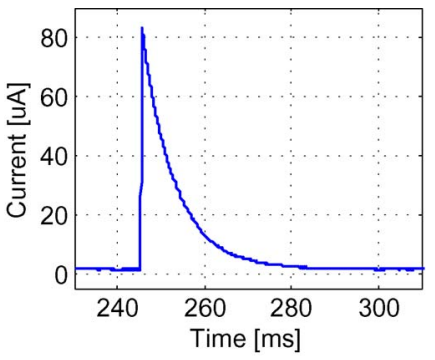

(b)
Fig. 18. Supply current waveforms for power-save-counters update-powersave transitions. Measurement range: $100 \mu \mathrm{A}$. (a) Test case-node only updates the clock. (b) Demo case-node in demo WSN updates clock and protocol timers.

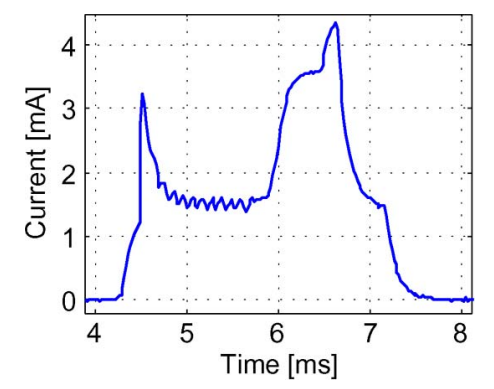

Fig. 19. Supply current waveform for sensing the channel. Measurement range: $10 \mathrm{~mA}$.

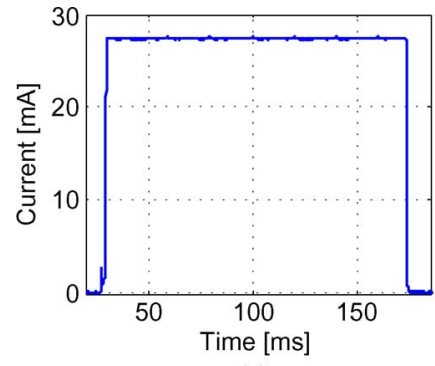

(a)

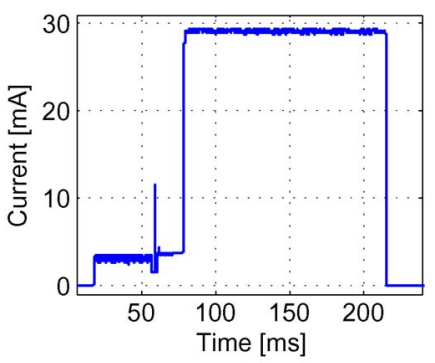

(b)
Fig. 20. Supply current waveforms for transmitting a frame. Measurement range: $100 \mathrm{~mA}$. (a) Test case-Tx for $136.6 \mathrm{~ms}$. (b) Demo case-Tx a frame.

the timers are responsible for an additional charge consumption of $174.5 \mathrm{pAh}$.

2) Channel Sensing: In order to sense the channel, the transceiver needs to get into reception mode and measure the received signal strength. Several nodes were measured, exhibiting the typical specified phase-locked loop lock times of $1.5 \mathrm{~ms}$. Fig. 19 shows the supply current waveform when sensing the channel which corresponds to an average of $2.09 \mathrm{nAh}$.

3) Frame Transmission: The transmission at $25 \mathrm{~kb} / \mathrm{s}$ for $136.6 \mathrm{~ms}$ requires an average of $1.09 \mu \mathrm{Ah}$ for the test case. In a running network (demo case), one should add the overhead related to the channel sensing and the random back-off interval of the CSMA-CA before transmitting. In this case, an average of $1.17 \mu \mathrm{Ah}$ was measured for sending a frame of $61 \mathrm{~B}$. Fig. 20(a) and (b) shows the current consumption waveforms during transmission for both investigated cases.

4) Frame Reception: A node in reception mode for $29.3 \mathrm{~ms}$ consumed, on average, $39.3 \mathrm{nAh}$ [test case-supply 


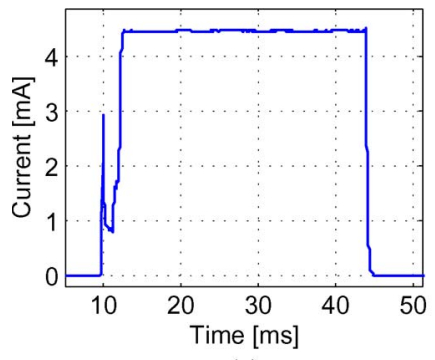

(a)

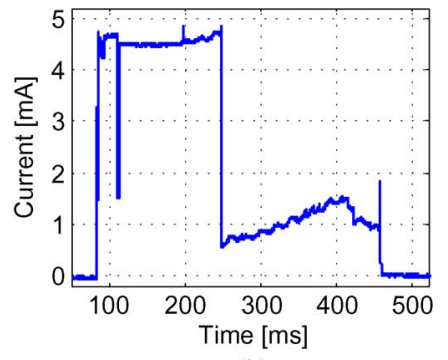

(b)
Fig. 21. Supply current waveforms for receiving a frame. Measurement range: $100 \mathrm{~mA}$. (a) Test case-Rx for $29.3 \mathrm{~ms}$. (b) Demo case-receiving a frame.

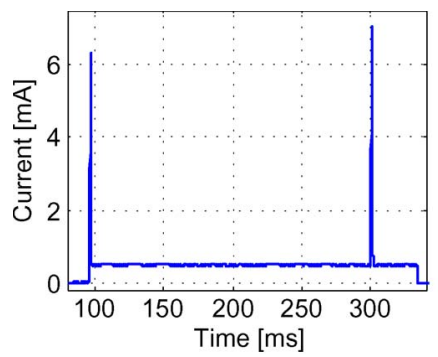

(a)

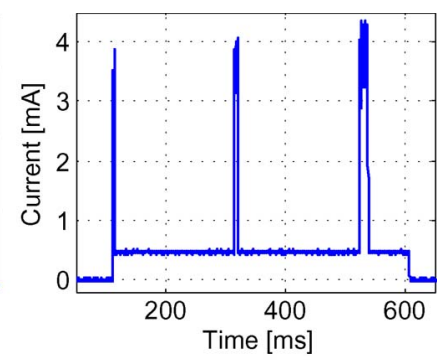

(b)
Fig. 22. Current waveforms for measuring the temperature with the DS7505 sensor. Measurement range: $10 \mathrm{~mA}$. (a) Single measurement. (b) Two measurements (demo case).

TABLE II

Measured Values for Charge Consumed on DifFerent TASKS FOR A NODE OPERATING IN A DEMO WSN

\begin{tabular}{l|c}
\hline \hline Task & {$[\mu \mathbf{A h}]$} \\
\hline \hline $1 \mathrm{~h}$ Power-save mode (timer active) & 1.62 \\
\hline $1 \mathrm{~h}$ of Clock \& counters updates & 0.63 \\
\hline $1 \times$ Channel sensing & 0.002 \\
\hline $1 \times$ Sending a frame & 1.17 \\
\hline $1 \times$ Receiving a frame & 0.24 \\
\hline $1 \times$ Temperature measuring & 0.08 \\
\hline \hline
\end{tabular}

current shown in Fig. 21(a)], while a node in the demo condition receives and processes a frame requiring, on average, $241 \mathrm{nAh}$ [Fig. 21(b)]. The difference in charge consumption is due to several additional tasks that the node in the demo WSN executes: After receiving the frame which takes, on average, only $29 \mathrm{nAh}$, the node blinks a LED (165 nAh), and the rest of the charge (approximately $47.6 \mathrm{nAh}$ ) is consumed for processing the received frame.

5) Temperature Measurement: The current waveforms for the temperature measurement are shown in Fig. 22(a) when running a test firmware for one measurement and in Fig. 22(b) when a node runs in the demo network (two measurements one after the other). The charge consumptions are 36.9 and $80.3 \mathrm{nAh}$ for one and two measurements, respectively. These values are comparable with the performances stated in the data sheet of the temperature sensor DS7505 (maximum of $750 \mu \mathrm{A}$ for $200 \mathrm{~ms}$ which is $41.7 \mathrm{nAh}$ ). For an easier comprehension, the measured charge consumptions in the demo case are summarized in Table II.
TABLE III

COMPUTED CONSUMPTION OF A NODE OPERATING FOR 70 DAYS IN A DEMO WSN

\begin{tabular}{l|l}
\hline \hline Task & {$[\mathbf{m A h}]$} \\
\hline \hline Power-save mode (timer active) & 2.72 \\
\hline Clock \& counters updates & 0.76 \\
\hline Sending a frame once per minute & 118 \\
\hline $\begin{array}{l}\text { Channel sensing 3s @ 100 ms } \\
\text { duty-cycle after each sent frame }\end{array}$ & 6.05 \\
\hline Temperature measuring & 8.06 \\
\hline Receiving a frame & 24.2 \\
\hline \hline Total & 159.79 \\
\hline
\end{tabular}

\section{Long-Term Battery Lifetime}

The demo WSN was used to evaluate the long-term battery lifetime. In order to mimic a real working scenario, seven sensor nodes were distributed throughout the offices of the Electronics and Signal Processing Laboratory, École Polytechnique Fédérale de Lausanne. The nodes (including one placed in a deep freezer at $-20^{\circ} \mathrm{C}$ ) were communicating with the gateway node which was wired to a PC running the WSN coordinator software. The system was left running with a duty cycle set to 1 min (each node performs a temperature measurement every minute and transmits the data to the gateway) until batteries are depleted. This condition represents a "busy" network operation, and it implies that a node receives approximately one frame for each frame it sends.

Two different power-supply configurations were considered while performing the test using the Renata CR2032 primary lithium battery (capacity of $225 \mathrm{mAh}$ ): a single battery for the nodes at room temperature and two batteries connected in parallel with the addition of a supercapacitor for the node in the freezer. The average long-term battery lifetime was 70 days for the single-battery nodes and 128 days for the double plus supercapacitor solution. The worse performance of the latter case (double capacity without twice the lifetime) is due to the increased internal resistance of the batteries at lower temperatures.

Extrapolating the information given in Section V-B, Table III reports the computed charge consumption per task of the node running in the demo WSN until battery depletion. This analysis shows that only approximately $70 \%$ of the battery capacity (225 mAh) is necessary for the 70-day lifetime period. The difference between the nominal charge of the battery and what was actually consumed is due to the retransmissions and external and mutual interferences, as well as battery self-discharge $(<1 \% / y e a r)$, that occur in a real scenario. It should be also noted that the nominal battery capacities are specified for discharge down to $2 \mathrm{~V}$ (not to $2.1 \mathrm{~V}$ ), at room temperature and at currents much lower than the one required for transmission.

These tests provide useful information for the power analysis of the RoSe node, particularly regarding the use of different duty cycles and/or power supplies. For instance, considering a $70 \%$ use of the nominal capacity and assuming $1 \%$ selfdischarge, extending the duty cycle to 15 min would result in a lifetime of approximately 4 years with two CR2032 coin cells and the supercapacitor and of 9 years with the $1 / 2$ AA battery. 
TABLE IV

SUMMARY AND COMPARISON OF THE ROSE NODE CHARACTERISTICS. SUPPly VOLTAGE: 3.6 V FOR [20] AND 3 V FOR THE NEW DESIGN

\begin{tabular}{|c|c|c|}
\hline \multicolumn{3}{|c|}{$\begin{array}{l}\text { Parameter Comparison Between [20] and the RoSe node both with } \\
\text { MCU clock of } 1.8432 \mathrm{MHz} \text {. }\end{array}$} \\
\hline Parameter & [20] & RoSe node \\
\hline Supply voltage range [V] & $2.7-3.6$ & $2.1-3.6$ \\
\hline Power-save current typ. $[\mu \mathrm{A}]$ & $2-4$ & $<2$ \\
\hline Rx current $[\mathrm{mA}]^{\mathrm{a}}$ & $\leq 15$ & $<5$ \\
\hline Tx current $[\mathrm{mA}]^{\mathrm{a}}$ & $>60$ & $<35$ \\
\hline RF chip Tx power [dBm] & Up to 15 & Up to 12.5 \\
\hline Antenna gain [dBi] & $<-10$ & -4 to -6 \\
\hline Bit rate [kbit/s] & Up to 76.8 & Up to 200 \\
\hline Program memory & 22 kB (8kInstr.) & $16 / 32 / 64 / 128 \mathrm{~KB}$ \\
\hline $\mathrm{I}^{2} \mathrm{C}$, SPI \& UART peripherals & Software & In Hardware \\
\hline PCB layers / surface $\left[\mathrm{cm}^{2}\right]$ & $4 / 25$ & $2 / 12.5$ \\
\hline Overall volume $\left[\mathrm{cm}^{3}\right]$ & $\approx 120$ & $\approx 72$ \\
\hline $\begin{array}{l}\text { Battery (lifetime at } 15 \mathrm{~min} . \\
\text { measurement interval) }\end{array}$ & $\begin{array}{c}\text { AA } 3.6 \mathrm{~V} \\
2250 \mathrm{mAh} \\
(3 \mathrm{y})\end{array}$ & $\begin{array}{c}1 / 2 \text { AA } 3.6 \mathrm{~V} \\
1100 \mathrm{mAh}(9 \mathrm{y}) \text { or } \\
2 \times \mathrm{CR} 20323 \mathrm{~V} \\
225 \mathrm{mAh}(4 \mathrm{y}) \\
\end{array}$ \\
\hline Enclosure protection & IP61 & IP66 \\
\hline
\end{tabular}

${ }^{\text {a }}$ Supply voltage $3.6 \mathrm{~V}$ for [20] and $3 \mathrm{~V}$ for the new design.

\section{Comparison With the Previous Platform}

In order to appreciate the properties of the RoSe node, Table IV compares its performances with the ones of the solution commercially available for the same application [20].

Given a similar voltage supply, the proposed platform has less than half of the current consumption in powersave mode, while the transmit (Tx) and receive ( $\mathrm{Rx})$ currents are reduced by a factor of two and three, respectively. Bit rate and programming memory are widely improved which result, together with the various possible power supplies, in a much more versatile solution. Indeed, the Semtech XE8806A used in [20] offers only 8000 instructions of program memory, and it takes approximately $2 \mathrm{~min}$ to perform the programming as compared to 8-64 $\mathrm{k}$ and less than $10 \mathrm{~s}$ of the RoSe MCU. Furthermore, smaller packaging sizes of the ICs allow for a smaller PCB having two instead of four layers. Finally, the realization of the integrated overmolded antenna presents several benefits: smaller overall volume, higher antenna performances (more than 5-dB gain increase), and improved enclosure protection (from IP61 to IP66 [22]).

\section{CONCLUSION}

We have presented a new wireless sensor platform working in the subgigahertz ISM band which introduces the novel concept of overmolded antenna housing. The produced node meets the requirements of the food cold chain management industry. The design process has followed a global approach considering each aspect constituting the node (electronics hardware, antenna housing, battery, and communication protocol) so as to realize a compact, ultralow-power, and physically robust node.

Injection molding technology has been selected to meet the requirements of the targeted application as it provides the required physical robustness and resistivity against humidity, and it is compatible with low-cost mass production. Therefore, overmolding investigations have been performed on both active and passive components. The experimental analysis on the former has shown that the MCU, peripheral components, and quartz-crystal frequency oscillators sustain the harsh conditions of the technological process, while the SAW filter and the quartz crystal for the radio transceiver required extra protection to retain their full functionality. The benefits of plastic injection molding have been also considered in the radiator conception reaching the design of a housing-integrated overmolded antenna. The proposed compact radiator fulfils EM, mechanical, industrial, and food compatibility requirements. Indeed, this antenna concept makes an efficient use of the available volume (thus, enhancing the EM radiation) and provides a robust enclosure protection which still maintains the accessibility to the batteries to further extend the lifetime of the node. Furthermore, the complete RoSe platform is a versatile solution (the same architecture for gateway/relay/sensor nodes and only minor modifications required to ensure the use in the U.S. ISM frequency spectrum) which makes it very appealing for a commercial application.

To evaluate the production stability, 15 nodes with antenna housings have been produced and tested. The measurement results presented in this paper reflect the characteristics obtained from this node population. The experimental analysis of the platform power consumption has been performed. The values obtained from the long-term battery lifetime measurements in the demo WSN are in good agreement with the calculations based on per-task charge consumption measurements. These results provide useful information regarding the use of different duty cycles and/or power supplies for an improved power management analysis.

In order to appreciate the characteristics of the proposed node, its characteristics have been compared to the ones of a commercially available solution for the very same application. The RoSe platform outperforms the previous node with production costs which are approximately reduced by a factor of three.

Future work includes firmware and protocol optimization to increase overall WSN reliability. Changes to the platform and the protocol in order to accommodate eventual new industrial or research applications are also being considered.

\section{ACKNOWLEDGMENT}

The authors would like to thank the industrial partners IP01 SA and TURCK duotec SA for their fruitful collaboration and contribution.

\section{REFERENCES}

[1] IEEE Standard for a Smart Transducer Interface for Sensors and Actuators-Mixed-Mode Communication Protocols and Transducer Electronic Data Sheet (TEDS) Formats, IEEE Std. 1451.4-2004, 2004, pp. 0_1-430.

[2] IEEE Standard for Information Technology-Telecommunications and Information Exchange Between Systems - Local and Metropolitan Area Networks-Specific Requirements Part 15.4: Wireless Medium Access Control (MAC) and Physical Layer (PHY) Specifications for Low-Rate Wireless Personal Area Networks (WPANs), IEEE Std. 802.15.4-2006, 2006, (Revision of IEEE Std 802.15.4-2003), pp. 0_1-305.

[3] P. Dutta, J. Taneja, J. Jeong, X. Jiang, and D. Culler, "A building block approach to sensornet systems," in Proc. 6th ACM Conf. Embedded Netw. Sens. Syst., 2008, pp. 267-280. 
[4] H. Dubois-Ferriere, R. Meier, L. Fabre, and P. Metrailler, "TinyNode: A comprehensive platform for wireless sensor network applications," in Proc. 5th Int. Conf. IPSN, 2006, pp. 358-365.

[5] R. V. Martinez-Catala and J. Barrett, "A modular wireless sensor platform with fully integrated battery," IEEE Trans. Compon. Packag. Technol., vol. 32, no. 3, pp. 617-626, Sep. 2009.

[6] L. Huang-Chen, A. Banerjee, F. Yao-Min, L. Bing-Jean, and K. Chung-Ta, "Design of a multifunctional wireless sensor for in-situ monitoring of debris flows," IEEE Trans. Instrum. Meas., vol. 59, no. 11, pp. 2958-2967, Nov. 2010.

[7] A. Carullo, S. Corbellini, M. Parvis, and A. Vallan, "A wireless sensor network for cold-chain monitoring," IEEE Trans. Instrum. Meas., vol. 58, no. 5, pp. 1405-1411, May 2009.

[8] C. C. Enz, A. El-Hoiydi, J. D. Decotignie, and V. Peiris, "WiseNET: An ultralow-power wireless sensor network solution," Computer, vol. 37, no. 8, pp. 62-70, Aug. 2004.

[9] R. Riem-Vis, "Cold chain management using an ultra low power wireless sensor network," in Proc. MobiSys, WAMES, 2004, pp. 21-23.

[10] M. Tanevski, A. Boegli, P. A. Farine, F. Merli, J. F. Zurcher, and A. Skrivervik, "Robust ultra low power wireless sensor platform with embedded over-molded antenna," in Proc. IEEE SAS, pp. 51-56.

[11] TURCK duotec SA. [Online]. Available: http://www.turck-duotec.com/ en/mechanical-packaging.html

[12] NXP I2C Specification and User Manual, NXP Semiconductors, Eindhoven, The Nederlands. [Online]. Available: http://www.nxp.com/ documents/user\%5Fmanual/UM10204.pdf

[13] SX1211 Ultra-Low Power Integrated UHF Transceiver Datasheet. [Online]. Available: http://www.semtech.com/images/datasheet/sx1211.pdf

[14] K. H. J. K. Fujimoto and A. Henderson, Small Antennas, 1st ed. Letchworth, England: Electron. Elect. Res. Stud. Press, 1987, ser. Antenna Series.

[15] "Agilent 85070e dielectric probe kit $200 \mathrm{MHz}$ to $50 \mathrm{GHz}$ technical overview," Agilent Technol., Santa Clara, CA, Tech. Rep. [Online]. Available: http://cp.literature.agilent.com/litweb/pdf/5989-0222EN.pdf

[16] J. F. Max Sucher, Handbook of Microwave Measurements, 3rd ed. Brooklyn, NY: Polytechnic Press PIB, 1963.

[17] Boedeker Polypropylene Specifications. [Online]. Available: http://www. boedeker.com/polyp_p.htm

[18] J. F. Zürcher, O. Staub, A. K. Skrivervik, and M. Hermanjat, "Accurate measurement of the maximum gain of electrically small antennas," Microw. Opt. Technol. Lett., vol. 23, no. 6, pp. 328-331, Dec. 1999.

[19] A. K. Skrivervik, J. F. Zürcher, O. Staub, and J. R. Mosig, "PCS antenna design: The challenge of miniaturization," IEEE Antennas Propag. Mag., vol. 43, no. 4, pp. 12-27, Aug. 2001.

[20] Temperature Sensor Product Datasheet. [Online]. Available: http://www. ip01.com/uploads/telechargements/6000-20x0-04en.pdf

[21] IP01 SA. [Online]. Available: http://www.ip01.com

[22] Degrees of Protection Provided by Enclosures (IP Code), IEC 60529 Ed.2.1, 2001, pp. 1-91.

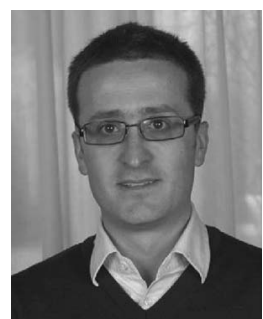

Francesco Merli (M'07) received the Laurea degree (cum laude) in telecommunication engineering from the University of Florence, Florence, Italy, in 2006 and the $\mathrm{Ph} . \mathrm{D}$. degree in electrical engineering from Ecole Polytechnique Fédérale de Lausanne (EPFL), Lausanne, Switzerland, in 2011.

$\mathrm{He}$ is currently with the Laboratory of Electromagnetics and Acoustics, EPFL. His research interests include antenna theory with particular focus on implantable and ultra-wide band antennas, spherical wave analysis, biomedical applications, wireless sensing, and atomic watch cavities.

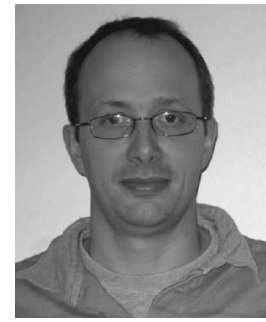

Alexis Boegli received the diploma in electronics physics and the Ph.D. degree from the University of Neuchâtel (UniNE), Neuchâtel, Switzerland, in 1995 and 2001, respectively.

Starting with a mechanical background, he has a 15 -year experience in the design and implementation of analog and digital electronic systems. Since 2001, he has focused on the design and implementation of RF systems. He has designed several RF boards for IP01 including the actually commercialized one. Being a Team Leader with the Electronics and Signal Processing Laboratory, Institut de Microtechnique, Neuchâtel, Switzerland (being at the UniNE until the end of 2008, the institute was then transferred at EPFL), he is challenged by the optimization of size, range, power, and production of wireless systems. He is also managing different medical technology projects and scavenging electronics research and development.

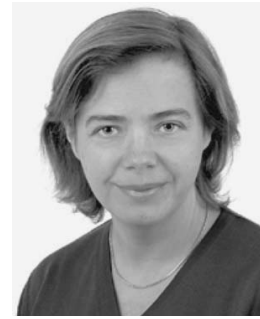

Anja K. Skrivervik received the electrical engineering degree and the Ph.D. degree from Ecole Polytechnique Fédérale de Lausanne (EPFL), Lausanne, Switzerland, in 1986 and 1992, respectively.

After a passage at the University of Rennes, Rennes, France, and the industry, she returned to EPFL as an Assistant Professor in 1996, where she is currently a "Professeur Titulaire." Her teaching activities include courses on microwaves and on antennas. Her research activities include electrically small antennas, multifrequency and ultrawideband

Mitko Tanevski (S'10) received the engineering diploma in electronics and telecommunications from Ss. Cyril and Methodius University, Skopje, Macedonia, in 2005. He is currently working toward the Ph.D. degree at Ecole Polytechnique Fédérale de Lausanne, Neuchâtel, Switzerland.

His present research interests are in wireless sensor networks (WSNs), small-footprint energyefficient communication protocols for WSNs, and energy management in embedded applications. antennas, and numerical techniques for electromagnetic and microwave and millimeter-wave microelectromechanical systems (MEMS). She is the author or coauthor of more than 100 scientific publications. She is very active in European collaboration and European projects.

Dr. Skrivervik is currently the Chairperson of the Swiss International Scientific Radio Union, the Swiss Representative for European cooperation in science and technology action 297, and a Member of the Board of the Center for High Speed Wireless Communications of the Swedish Foundation for Strategic Research. 


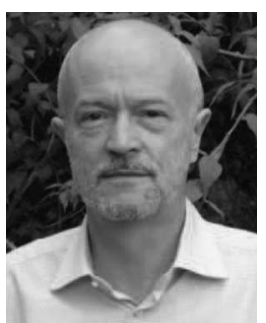

Jean-François Zürcher was born in Vevey, Switzerland, in 1951. He received The degree of Electrical Engineer from Ecole Polytechnique Fédérale de Lausanne (EPFL) (Lausanne Institute of Technology), Lausanne, Switzerland, in 1974.

$\mathrm{He}$ is currently a Permanent Scientific Associate with the Laboratory of Electromagnetics and Acoustics, EPFL, where he is the Manager of the microwave laboratory. His main interest lies in the domain of microstrip circuits and antennas. In 1988, he invented the "strip slot foam inverted patch antenna" concept, which became a commercial product. He is presently developing instrumentation and techniques for the measurement of near fields of planar structures and microwave materials measurement and imaging. He is the author or coauthor of about 125 publications, chapters in books, and papers presented at international conferences. He is one of the two authors of the book "Broadband Patch Antennas" (Artech, 1995). He is the holder of eight patents.

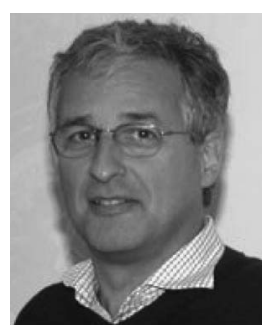

Pierre-André Farine (M'85-M'95) received the Engineering degree in microtechnology from Haute école spécialisée (HES) [formerly école technique supérieure (ETS)], Le Locle, Switzerland, in 1974 and the M.S. and Ph.D. degrees in microtechnology from the University of Neuchâtel (UniNE), Neuchâtel, Switzerland, in 1978 and 1984, respectively.

For 17 years, he was with the Swatch Group R\&D laboratories for consumer wearable low-power products, for technical watches such as Tissot "T-Touch" or Longines "Very High Precision." Since 2002, he has been a Full Professor with the Institute of Microtechnology, UniNE, leading the Electronics and Signal Processing Laboratory, specialized for low-power CMOS integrated circuits for applications covering neuroscience, wireless sensor networks, global navigation satellite system and UWB telecommunications, and video and audio processing. Since 2009, he has been a Full Professor with Ecole Polytechnique Fédérale de Lausanne (EPFL), Neuchâtel, and his laboratory has been transferred from UniNE to EPFL. He authored more than 100 publications in conferences and in scientific journals and is the holder of 50 patent families.

Dr. Farine has served as a member of program committees and Program Chair for international conferences. 